\title{
eMOC: Ferramenta Para o Mapeamento Através da Observação do Comportamento de Alunos Cegos na Sala de Aula
}

\author{
Bruna Lima $^{3}$, Angelica F. S. Dias ${ }^{1,2}$, Juliana B. S. França ${ }^{1}$, Marcos R.S. Borges ${ }^{13 ;}$ José Antônio $^{2}$ \\ S. Borges ${ }^{2,4}$ \\ ${ }^{1}$ Programa de Pós-graduação em Informática/PPGI(UFRJ) \\ ${ }^{2}$ Instituto Tércio Pacitti da Universidade Federal do Rio de Janeiro (UFRJ) \\ ${ }^{3}$ Departamento de Ciência da Computação / UFRJ \\ ${ }^{4} \mathrm{HCTE}$ - História das Ciências e das Técnicas e Epistemologia (UFRJ) \\ limacosta25@dcc.ufrj.br ; \{angelica, juliana.frança, mborges\}@ppgi.ufrj.br, \{antonio2\}@nce.ufrj.br
}

\begin{abstract}
Interaction problems among students often occur while performing group tasks in classrooms. This can lead to the loss of knowledge and reduce the efficiency of the workgroup. In this context, this work proposes the use of a computational tool to support observations that, in a collaborative way, allow teachers to identify cognitive barriers among students while performing group tasks in the classroom. Our aim is to provide teachers with mechanisms that facilitate the transfer of knowledge among students, especially blind students, while performing group tasks.
\end{abstract}

Resumo. Problemas de interação entre os alunos muitas vezes ocorrem durante a execução de tarefas em grupo nas salas de aula. Isto pode levar à perda de conhecimento e à baixa eficiência do grupo de trabalho. Nesse contexto, este trabalho propõe o uso de uma ferramenta computacional para apoiar observações que, de forma colaborativa, permitem aos professores identificar barreiras cognitivas entre os alunos durante a realização de tarefas na sala de aula. Espera-se fornecer aos professores mecanismos que facilitem a transferência de conhecimento entre alunos, especificamente alunos cegos, na realização de trabalho em grupo.

\section{Introdução}

As ferramentas tecnológicas permitem, no contexto educacional, aumentar as possibilidades para o desenvolvimento de práticas contemporâneas, a partir de mecanismos que garantam o acompanhamento e interação entre os alunos. Choing e Jovanovic (2012) apresentam uma abordagem para promover a aprendizagem colaborativa através da interação entre os indivíduos para realização de suas atividades na sala de aula. Contudo, este processo necessita de uma análise dos indivíduos, seus comportamentos e os elementos que influenciam os resultados do grupo.

Friard e Gamba (2016) apresentam em suas pesquisas investigações realizadas a partir de coletas de dados, através de observações, onde afirmam que somente a análise dos dados quantitativos podem influenciar os resultados do trabalho em grupo. Estas técnicas utilizam muitas informações, onde partes destes dados são generalizados em campos como os da psicologia, antropologia, neurociência e ciência cognitiva (Cristiani et al 2013). Considerando que a pesquisa apoiada pela observação comportamental possibilita o surgimento de ferramentas para a análise quantitativa. Este trabalho tem 
VII Congresso Brasileiro de Informática na Educação (CBIE 2018)

Anais do XXIX Simpósio Brasileiro de Informática na Educação (SBIE 2018)

como objetivo fornecer aos professores mecanismos que facilitem a transferência de conhecimento através de dados coletados durante 0 ato de observação do comportamento dos alunos, especificamente alunos cegos, na realização de tarefas nos grupos de trabalho nas salas de aula.

Para esta pesquisa utilizamos o método de observação direta estruturada. Nela os dados da observação são detalhados em um sistema colaborativo considerando contexto de sala de aula. É na ferramenta colaborativa onde podemos observar a troca de conhecimento tácito entre os alunos e professores, e extrair seus comportamentos através de eventos e situações reais (Pimentel e Fuks, 2011). As estratégias para observação e aplicação do método são apresentadas em um experimento piloto, onde são destacadas as características da ferramenta e, identificados fragmento de interações entre os alunos.

\section{Aprendizagem Colaborativa e a Observação do Comportamento do Aluno}

Para esta pesquisa utilizamos a Aprendizagem Colaborativa com Suporte Computacional (Computer Supported Collaborative Learning -CSCL), que é um ramo emergente das ciências da aprendizagem que estuda como as pessoas podem aprender em grupo com o auxílio do computador. Para este estudo, o aluno é um agente ativo no processo de aprendizagem, que interage com os outros, assimilando conceitos e construindo conhecimento (Barcelos, 2012). A aprendizagem é um campo teórico que compreende o processo eminentemente de trocas sociais (Arruda et al, 2017). Esta abordagem está fundamentada nos pressupostos da teoria sociocultural de Vygostky (2002), onde os elementos sociais podem interagir com os tecnológicos e apoiar o processo de aprendizagem.

Estudos apresentam diversas alternativas para a produção de materiais tecnológicos que forneçam suporte à aprendizagem, apoiando os professores no planejamento de suas atividades (Castro, 2007; Faria 2003). Considerando esse contexto e as dificuldades mencionadas, o trabalho propõe uma abordagem colaborativa suportada por uma ferramenta de observação sobre o comportamento dos indivíduos, mapeando a interação entre os alunos durante a transferência de conhecimento.

Para Friard e Gamba (2016), muitas pesquisas sobre observação de grupos lidam com ferramentas tecnológicas para o uso de acessos remoto sobre uma quantidade de dados, análise e monitoramento do comportamento dos indivíduos. No entanto, estes sistemas são caros e complexos, necessitando de um desenvolvimento em conjunto de softwares comerciais com muitas limitações de recursos analíticos (Noldus et al. 2015). Além disso, não apresentam funcionalidades de colaboração, que para esta pesquisa é considerado fundamental.

Esta pesquisa propõe, a condução da observação direta estruturada através do suporte tecnológico da ferramenta eMOC - Mapeamento Através da Observação do Comportamento do Especiaslistas (Professores) e Aprendizes (Alunos), conforme Figura 1. Esta aplicação foi desenvolvida em Python3, para Microsoft Windows. Ela tem o objetivo de mapear o comportamento de indivíduos que compõem grupos, através da observação de vídeos que retratam cenários reais. 
VII Congresso Brasileiro de Informática na Educação (CBIE 2018)

Anais do XXIX Simpósio Brasileiro de Informática na Educação (SBIE 2018)

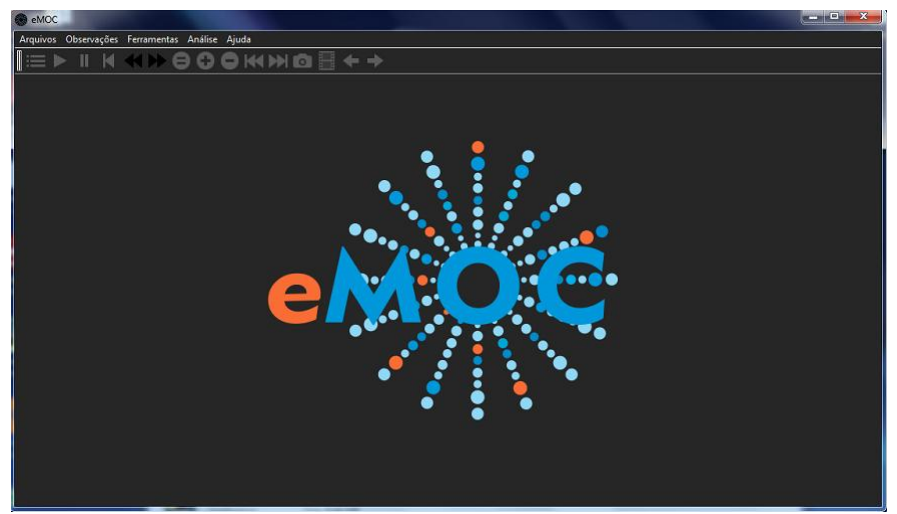

Figura 1: Tela Inicial do eMOC.

O eMOC é uma ferramenta capaz de gerar cenários de um número ilimitado de eventos e assuntos ocorridos ao longo de situações cotidianas. A ferramenta também permite extrair informações de eventos simples ou agrupados de forma colaborativa. Para a ferramenta eMOC, um conjunto de professores podem gerir um status de tempo e apresentar um resumo das últimas análises realizadas das atividades do grupo de trabalho, bem como de cada aluno. Com isso, elementos implícitos existentes nas interações entre os indívíduos, ao serem observados, podem ser identificados formando grupos mais eficientes.

\section{Experimento}

Para atender ao nosso objetivo, organizamos um estudo piloto, através do ensino da matemática para alunos cegos, com baixa visão ou visão normal, da engenharia da Computação de uma universidade federal. Este estudo foi conduzido por um professor, da disciplina de computação gráfica, no Laboratório de Tecnologia Assistiva, NCE/UFRJ.

Para este estudo foi utilizado o Geoplano (Figura 2) - objeto de aprendizagem para o ensino de geometria - para apoiar e tornar tangível o plano geométrico. $\mathrm{O}$ experimento foi composto de duas fases. Na primeira fase, foi realizado um exercício de geometria em grupo, onde os alunos foram apresentados aos elementos matemáticos. Durante o experimento, foram observadas as interações dos alunos com o professor e identificadas possíveis barreiras positivas e negativas no processo de transferência do conhecimento no aprendizado da matemática. Na segunda fase, os vídeos gerados durante o experimento foram analisados através da ferramenta eMOC (Figura 3). Com os resultados alcançados nas análises, foi possível sugerir melhorias ao método de aprendizagem adotado com os alunos. 
VII Congresso Brasileiro de Informática na Educação (CBIE 2018)

Anais do XXIX Simpósio Brasileiro de Informática na Educação (SBIE 2018)

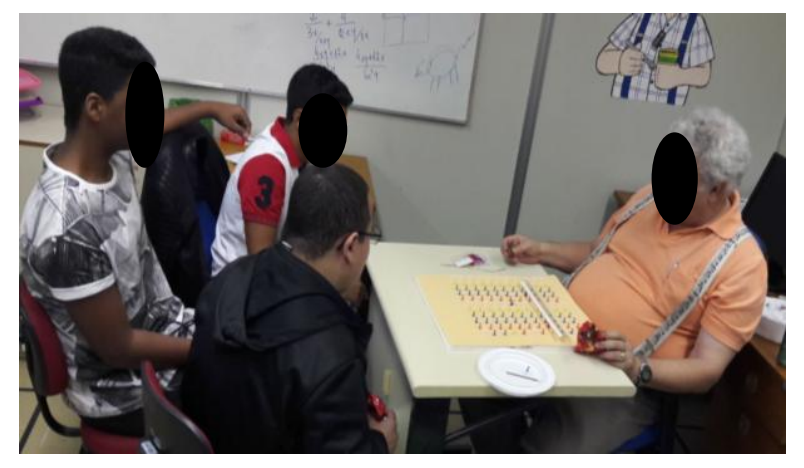

Figura 2: Etapa: Atividade do grupo com Geoplano

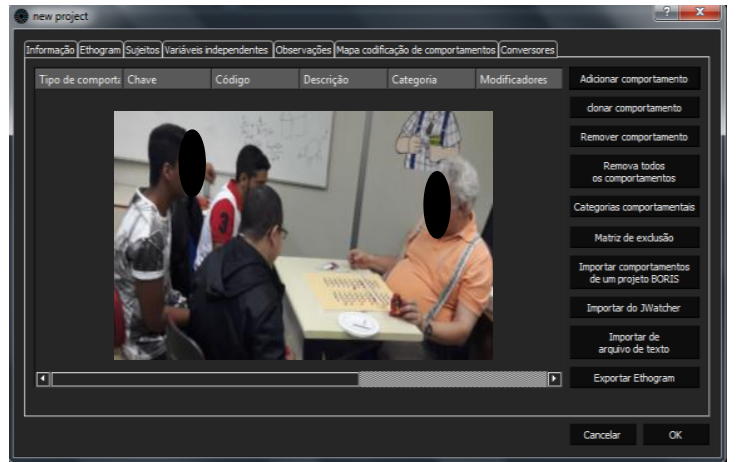

Figura3: Observação e Análise do experimento

\section{Conclusões e Próximos Passos}

Este artigo apresentou a ferramenta eMOC, pois trata-se de um projeto de pesquisa com foco na observação da interação entre professor e alunos, principalmente alunos cegos, para apoiar o ensino da matemática na sala de aula. Com os resultados alcançados no projeto piloto, iniciamos ajustes e melhorias no desenvolvimento da ferramenta. Além de incluir novas funcionalidades geradas pelas análises das observações e resultados dos indivíduos envolvidos no experimento. Como etapas futuras, destacamos: (i) conclusão do desenvolvimento da aplicação; (ii) realização de testes para validar o método e a ferramenta; e (iii) a realização de um novo experimento com um conjunto maior de alunos em outras instituições públicas. Pretendemos com isso, avaliar o impacto e usabilidade da ferramenta e apoiar a inclusão de mais alunos com deficiência visual nas universidades públicas.

\section{Agradecimentos}

CNPq - CNPQ - Projeto Aceleração da Transferência de Conhecimento de Especialistas para equipes/Linha 2.

Laboratório de Tecnologia Assistiva, do Instituto Tércio Pacitti de Aplicações e Pesquisas Computacionais, da Universidade Federal do Rio de Janeiro. Sociedade Brasileira de Computação - Chancela SBC.

\section{Referências}

Arruda, J.S.; Filho, J.A.C.; Siqueira, L.M.R.C.; Hitzschky, R.A.; Brito, M.A.F. Tecnologias digitais e o processo de protagonismo estudantil no Ensino Fundamental. VI Congresso Brasilerio de Informática na Educação, Anais do XXIII Workshop de Informática na Escola (WIE 2017). Recife - PE

Barcelos, R. J. S. (2012) "O processo de construção do conhecimento de algoritmos com o uso de dispositivos móveis considerando estilos preferenciais de aprendizagem" Tese de Doutorado, Universidade Federal do Rio Grande do Sul (UFRGS).

Borges, J.A. Do Braille ao DOSVOX - diferenças nas vidas dos cegos brasileiros - Rio de Janeiro: UFRJ/COPPE, 2009. 
VII Congresso Brasileiro de Informática na Educação (CBIE 2018)

Anais do XXIX Simpósio Brasileiro de Informática na Educação (SBIE 2018)

Boulic, R. and Renault, O. (1991) "3D Hierarchies for Animation", In: New Trends in Animation and Visualization, Edited by Nadia Magnenat-Thalmann and Daniel Thalmann, John Wiley \& Sons 1td., England.

Chiong, R.; Jovanovic, J. Collaborative learning in online study groups: An evolutionary game theory perspective. Journal of Information Technology Education: Research, v.11, n.1, p.81$101,2012$.

Dias, A. F. S.; Franca, J.B.S.; Borges, M. R. S. Silva. Tecnologia Assistiva: Um Survey com portadores de deficiência visual em ambiente virtual de aprendizagem a partir do Modelo TAM. In: XVIII Conferência Internacional sobre Informática na Educação, TISE 2013, Porto Alegre

Dyer, S., Martin, J. and Zulauf, J. (1995) "Motion Capture White Paper", http://reality.sgi.com/employees/jam_sb/mocap/MoCapWP_v2.0.html, December.

Friard, O., Gamba, M. BORIS: a free, versatile open-source event-logging software for video/audio coding and live observations. Methods in Ecology and Evolution 2016, 7, 13251330. British Ecological Society.

Holton, M. and Alexander, S. (1995) "Soft Cellular Modeling: A Technique for the Simulation of Non-rigid Materials", Computer Graphics: Developments in Virtual Environments, R. A. Earnshaw and J. A. Vince, England, Academic Press Ltd., p. 449-460.

Knuth, D. E. (1984), The TeXbook, Addison Wesley, $15^{\text {th }}$ edition.

Noldus. The Observer XT ReferenceManual v. 12.5 www.noldus.com. 2015

Pimentel, M.; Fulks, H. Sistemas Colaborativos. Ed. Elsevier, 2011.

Smith, A. and Jones, B. (1999). On the complexity of computing. In Advances in Computer Science, pages 555-566. Publishing Press.

Vygotsky, L. S. (2002). A Formação Social da Mente. 6a. Edição. São Paulo: Martins Fontes. 